\title{
Burn trauma in skeletal muscle results in oxidative stress as assessed by in vivo electron paramagnetic resonance
}

\author{
NADEEM KHAN ${ }^{1}$, SRIRAM P. MUPPARAJU ${ }^{1}$, DIONYSSIOS MINTZOPOULOS ${ }^{2,3,5}$, MEENU KESARWANI ${ }^{4,5}$, \\ VALERIA RIGHI ${ }^{2,3}$, LAURENCE G. RAHME ${ }^{4,5}$, HAROLD M. SWARTZ ${ }^{1}$ and A. ARIA TZIKA ${ }^{2-5}$ \\ ${ }^{1}$ EPR Center for Viable Systems, Department of Diagnostic Radiology, Dartmouth Medical School, Hanover, NH 03755;
${ }^{2}$ Athinoula A. Martinos Center of Biomedical Imaging, Department of Radiology, Massachusetts General Hospital,
${ }^{3}$ NMR Surgical Laboratory, Department of Surgery, ${ }^{4}$ Molecular Surgery Laboratory, Department of Surgery, ${ }^{5}$ Department
of Surgery, Massachusetts General Hospital and Shriners Burn Institute, Harvard Medical School, Boston, MA 02114, USA
}

Received June 26, 2008; Accepted August 1, 2008

DOI: $10.3892 / \mathrm{mmr} 00000033$

\begin{abstract}
Using a mouse model, we tested the hypotheses that severe burn trauma causes metabolic disturbances in skeletal muscle, and that these can be measured and repeatedly followed by in vivo electron paramagnetic resonance (EPR). We used a 1.2-GHz (L-band) EPR spectrometer to measure partial pressure of oxygen $\left(\mathrm{pO}_{2}\right)$ levels, redox status and oxidative stress following a non-lethal burn trauma model to the left hind limbs of mice. Results obtained in the burned mouse gastrocnemius muscle indicated a significant decrease in tissue $\mathrm{pO}_{2}$ immediately $(\mathrm{P}=0.032)$ and at $6 \mathrm{~h}$ post burn $(\mathrm{P}=0.004)$, compared to the gastrocnemius of the unburned hind limb. The redox status of the skeletal muscle also peaked at $6 \mathrm{~h}$ post burn $(\mathrm{P}=0.027)$ in burned mice. In addition, there was an increase in the EPR signal of the nitroxide produced by oxidation of the hydroxylamine $(\mathrm{CP}-\mathrm{H})$ probe at $12 \mathrm{~h}$ post burn injury, indicating a burn-induced increase in mitochondrial reactive oxygen species (ROS). The nitroxide signal continued to increase between 12 and $24 \mathrm{~h}$, suggesting a further increase in ROS generation post burn. These results confirm genomic results, which indicate a downregulation of antioxidant genes and therefore strongly suggest the dysfunction of the mitochondrial oxidative system. We believe that the direct measurement of tissue parameters such as $\mathrm{pO}_{2}$, redox and ROS by EPR may be used to complement measurements by nuclear magnetic resonance (NMR) in order to assess tissue damage and the therapeutic effectiveness of antioxidant agents in severe burn trauma.
\end{abstract}

Correspondence to: Dr A. Aria Tzika, NMR Surgical Laboratory, Department of Surgery, Massachusetts General and Shriners Hospitals, Harvard Medical School, 51 Blossom Street, Room 261, Boston, MA 02114, USA

E-mail: atzika@hms.harvard.edu

Key words: electron paramagnetic resonance, oximetry, partial pressure of oxygen, redox, skeletal muscle, burn, trauma, mitochondria, reactive oxygen species, nuclear magnetic resonance, genomics

\section{Introduction}

Electron paramagnetic resonance (EPR) is a magnetic resonance-based technique that detects species with unpaired electrons, such as free radicals (organic or inorganic) and transition metal ions. The development of low frequency (1200 MHz and below) EPR spectrometers has led to the in vivo application of the technique in a variety of animal models. The most extensive applications of in vivo EPR have been repeated non-invasive measurements of oxygen, nitric oxide, free radicals, $\mathrm{pH}$ and tissue redox status (1-9). EPR in vivo has significantly contributed to the understanding of various pathological changes, and has the potential to become an important clinical tool $(4,9)$. Often, it may also be complementary to nuclear magnetic resonance (NMR) (1).

NMR is a useful method for measuring biomarkers in vivo, and has already shown relevance in the study of the metabolism and bioenergetics of skeletal muscle (10-25). In addition to NMR spectroscopy, the development of highthroughput microarray systems, capable of simultaneously measuring the expression of thousands of genes, has also greatly advanced our ability to detect and measure biomarkers. By exploring the expression of certain organ-specific candidate genes, the biological relevance of NMR data can be validated in microarray experiments examining the same specimen $(22,23,25)$. For assessing oxidative damage after burn trauma, in vivo EPR using nitroxides is complementary to NMR since NMR cannot measure redox status and reactive oxygen species (ROS), while EPR can.

Here, for the first time, we report tissue partial pressure of oxygen $\left(\mathrm{pO}_{2}\right)$, redox status and $\mathrm{ROS}$ measurement by in vivo EPR in intact proximal skeletal muscle tissue following burn trauma in mice. The potential significance of our findings include the in vivo non-invasive nature of the EPR measurements, which can serve to follow tissue pathology and to monitor the effectiveness of antioxidant agents in order to alleviate the symptoms of severe burn trauma. With the development and application of in vivo EPR oximetry in clinical settings $(4,9)$, its potential application in the clinical management of burn injury, alongside NMR, might also prove to be very useful. 


\section{Materials and methods}

Study design. Due to the in vivo repetitive nature of EPR measurements, the EPR experiments followed a longitudinal random-effects study design. This is powerful since each mouse contributed information at each of the time points, and the clustered data were modeled by a compound symmetry correlation structure (26). The genomics followed a crosssectional study design.

Experimental animals. Male 6-week-old CD1 mice weighing 20-25 g were purchased from the Charles River Laboratory (Boston, MA). The animals were maintained on a regular light-dark cycle (lights on from 8:00 to 20:00 h) at an ambient temperature of $22 \pm 1^{\circ} \mathrm{C}$ and had free access to food and water. All animal experiments were approved by the Subcommittee on Research Animal Care of the Massachusetts General Hospital, Boston, MA, and by the Institutional Animal Care and Use Committee of Dartmouth Medical School, Hanover, NH.

Hind limb burn model. Mice were anesthetized by intraperitoneal (i.p.) injection of $40 \mathrm{mg} / \mathrm{kg}$ pentobarbital sodium and were randomized into burn or control groups. The left hind limb of all mice in both groups (control and burn) was shaved, and each mouse in the burn group was subjected to a nonlethal scald injury of 3-5\% total body surface area (TBSA) by the immersion of its left hind limb in $90^{\circ} \mathrm{C}$ water for $3 \mathrm{sec}$, as previously described (27). The TBSA of each mouse was calculated using Meeh's formula: $\mathrm{A}=\mathrm{kx} \mathrm{W}^{2 / 3}$, where $\mathrm{A}$ is the surface area in $\mathrm{cm}^{2}, \mathrm{k}$ is a proportionality constant of 12.3 , and $\mathrm{W}$ is the weight of the mouse in grams. Mice were resuscitated with $2 \mathrm{ml}$ of $0.9 \%$ saline i.p. The gastrocnemius muscle was excised from the hind limbs of both control and treated mice and was immersed in $1 \mathrm{ml}$ TRIzol ${ }^{\circ}$ (GibcoBRL, Invitrogen, Carlsbad, CA) for RNA extraction at $6 \mathrm{~h}, 12 \mathrm{~h}$, 1 day and 3 days post burn, with $n=3$ measurements at each time point. After injury, the animals were given analgesia in the form of buprenorphine $0.05-0.1 \mathrm{mg} / \mathrm{kg} \mathrm{SQ}$, as needed.

Non-invasive in vivo EPR. EPR measurements were carried out with a 1.2-GHz EPR spectrometer equipped with a microwave bridge and external loop resonator specially designed for in vivo experiments. The optimal spectrometer parameters were: incident microwave power, $10 \mathrm{~mW}$; magnetic field center, 400 gauss; modulation frequency, $27 \mathrm{kHz}$. Modulation amplitude was one-third of the EPR line width, with a scan time of $10 \mathrm{sec}$. The methods described here are well established and routinely used in the EPR Center for Viable Systems at Dartmouth Medical School, Hanover, NH (1-9).

Measurement of $\mathrm{pO}_{2}$ using in vivo EPR. Repeated non-invasive measurements of the tissue $\mathrm{pO}_{2}$ of the gastrocnemius muscle of 4 control mice and 4 burned mice were carried out using in vivo EPR oximetry. Approximately 80-100 $\mu \mathrm{g}$ of sterilized EMS char (charcoal powder) was injected in the gastrocnemius muscle of the left hind limb of each mouse using an 18-gauge needle and plunger. The mice were allowed to recover for one week after char implantation. Once implanted, the paramagnetic material remains at the site and allows repeated tissue $\mathrm{pO}_{2}$ measurements without further invasion $(3,4,28)$.
For in vivo EPR oximetry, mice were anesthetized by $1.5 \%$ isoflurane with $30 \% \mathrm{O}_{2}$ and were gently placed in the magnet of the 1.2-GHz EPR spectrometer for $\mathrm{pO}_{2}$ measurements. Rectal temperature was continuously monitored and maintained at $37 \pm 1^{\circ} \mathrm{C}$ using a warm air blower and warm water pad. After a 30-min baseline measurement (pre-burn), the left hind limb of the mouse was exposed to hot water $\left(90^{\circ} \mathrm{C}\right)$ for $3 \mathrm{sec}$. For control experiments, the left hind limbs of the mice were exposed to $37^{\circ} \mathrm{C}$ water for $3 \mathrm{sec}$. The mice were immediately transferred back to the EPR magnet, and the tissue $\mathrm{pO}_{2}$ was measured for another 30 min (post-burn $\mathrm{pO}_{2}$ ) and at time points as indicated in the results.

Redox status measurements using nitroxides by in vivo EPR. Nitroxides exist in biological systems as a redox pair, namely the nitroxide free radical form and the diamagnetic hydroxylamine (one-electron reduction product of the nitroxide free radical). Nitroxides are reduced to the corresponding hydroxylamine in cell suspensions as well as in vivo by cellular reducing equivalents. Consequently, the pharmacology of nitroxides reports the redox status of the tissue $(7,29,30)$. Mice were anesthetized using $1.5 \%$ isoflurane with $30 \% \mathrm{O}_{2}$ delivered through a nose cone. The animals were placed on the warm water pad, and the tail vein was cannulated with a heparin-filled 30-gauge catheter for infusion of the nitroxide probe (3-carbamoyl-2,2,5,5-tetramethylpyrrolidin-1-yloxyl free radical, Sigma-Aldrich). The nitroxide $(150 \mathrm{mg} / \mathrm{kg}$ ) was injected slowly (over $1 \mathrm{~min}$ ) through the tail vein cannula. The left hind limb of the mice was immediately exposed to $90^{\circ} \mathrm{C}$ water for $3 \mathrm{sec}$, and the mice were quickly moved to the EPR magnet and positioned for the measurement of the signal intensity of the injected nitroxide in the gastrocnemius muscle of the left hind limb over time (Fig. 2A). The time difference between the infusion of the nitroxide and the start of EPR acquisition was $2 \mathrm{~min}$. This time was maintained in all the experiments. The change in signal intensity of the middle component of the nitroxide was followed over time (Fig. 2B), and data were fitted using exponential decay kinetics (Fig. 3) to determine the rate constants. For control experiments, the hind limb was exposed to $37^{\circ} \mathrm{C}$ water for $3 \mathrm{sec}$, and the measurements were repeated at identical time points. The same procedure was followed to measure the decay kinetics of the injected nitroxide at various time points, which indicated the redox status of the muscle.

ROS measurement using nitroxides by in vivo EPR spectroscopy. We measured ROS using EPR spectroscopy with a spin trapping technique that has previously been used to detect ROS production in vivo (31). The upregulation or downregulation of ROS was measured using a cyclic 1-hydroxy-3carboxy-2,2,5,5-tetramethylpyrrolidine probe $(\mathrm{CPH}$, Alexis Biochemicals). This EPR inactive probe is oxidized to the corresponding nitroxide $\left(\mathrm{CP}^{*}\right)$ by ROS. The increase in the EPR signal intensity of the nitroxide provides an indirect measure of the ROS in a tissue. Optimized spectrometer parameters and procedures as described above were used for these experiments. The $\mathrm{CPH}$ probe $(50 \mathrm{mg} / \mathrm{kg})$ was injected intravenously and the formation of the $\mathrm{CP} \cdot$ was followed over time by the placement of the EPR resonator over the gastrocnemius muscle. The increase in the EPR signal intensity of 


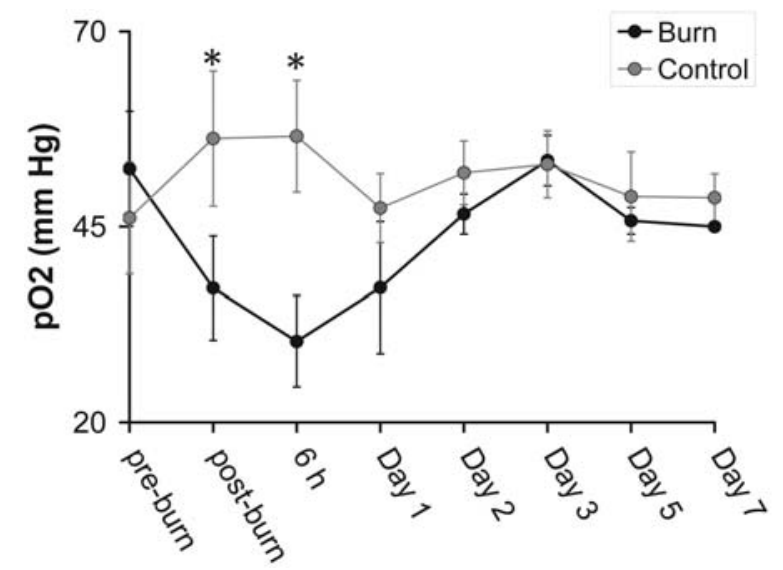

Figure 1. Repeated in vivo tissue $\mathrm{pO}_{2}$ measurements of the gastrocnemius skeletal muscle of the mice using EPR oximetry pre and post burn trauma at different time points. The post-burn data were acquired immediately after the burn followed by measurements at $6 \mathrm{~h}$ and on days 1-7. Values are the means $\pm \mathrm{SE} ;{ }^{*} \mathrm{P}<0.05, \mathrm{n}=4$.

the oxidized probe over time indicated the formation of ROS in the gastrocnemius muscle of the control and burn mice.

RNA extraction. At $6 \mathrm{~h}, 1$ day and 3 days post burn, 3 burned and 3 control mice were anesthetized at each time point by i.p. injection of $40 \mathrm{mg} / \mathrm{kg}$ pentobarbital, and the gastrocnemius was excised. All mice were then administered a lethal dose of pentobarbital (200 mg/kg i.p.), while the gastrocnemius muscle was isolated and immediately immersed in $1 \mathrm{ml}$ TRIzol for RNA isolation. The muscle was homogenized for $60 \mathrm{sec}$ using a Brinkmann Polytron PT 3000 homogenizer (Brinkmann Instruments, Westbury, NY) before the extraction of total RNA. Chloroform $(200 \mu \mathrm{l})$ was added to the homogenized muscle and mixed by inverting the tube for $15 \mathrm{sec}$. After centrifugation at $12,000 \mathrm{xg}$ for $15 \mathrm{~min}$, the upper aqueous phase was collected and precipitated by adding $500 \mu 1$ isopropanol. Further centrifugation at $12,000 \mathrm{x} g$ for $10 \mathrm{~min}$ separated the RNA pellet, which was then washed with $500 \mu 170 \%$ ethanol and centrifuged at 7,500 $\mathrm{x}$ g for $5 \mathrm{~min}$ prior to air-drying. The pellet was re-suspended in $100 \mu 1$ DEPC-H20. An RNeasy Kit (Qiagen, Valencia, CA) was used to purify the RNA according to the manufacturer's protocol. Purified RNA was quantified by UV absorbance at 260 and $280 \mathrm{~nm}$ and stored at $-70^{\circ} \mathrm{C}$ for DNA microarray analysis.

Microarray hybridization. Biotinylated cRNA was generated with $10 \mu \mathrm{g}$ of total cellular RNA according to the protocol outlined by Affymetrix Inc. (Santa Clara, CA). The cRNA was hybridized onto MOE430A oligonucleotide arrays (Affymetrix), stained, washed and scanned according to Affymetrix protocol.

Genomic data analysis. The scanned images of cRNA hybridization were converted to cell intensity files (CEL files) with the Microarray suite 5.0 (MAS, Affymetrix). Data were scaled to a target intensity of 500 , and for each time point all possible pair-wise array comparisons of the replicates to control mice were carried out (i.e., four combinations when the two arrays from each time point were compared with the two arrays from control mice) using a MAS 5.0 change call algo- rithm. Probe sets that had a signal value difference $\geq 100$ and in which both samples were present were scored as differentially modulated when i) the number of change calls in the same direction were at least 3,4 and 6 when the number of comparisons were 4, 6 and 9, respectively, and ii) the other comparisons were unchanged. Such scoring was to partially compensate for biological stochasticity and technical variation. Based on the ratios of 100 genes determined to be invariant in most of the conditions tested (Affymetrix) in the hind limb of burn and control animals, an additional constraint of a minimum ratio of 1.65 was applied to control the known false positives at $5 \%$ hind limb.

Statistical analysis. The Kolmogorov-Smirnov and Levene's tests were used to assess the normality and homogeneity of variances, respectively. Differences between control and burn groups were evaluated at each time point using twoway repeated-measures ANOVA (32) with time and group as factors for $\mathrm{pO}_{2}$, and by the Student's t-test for the rest. Statistical analysis was conducted using SPSS, version 15.0 (SPSS, Chicago, IL), and a two-tailed $\alpha$ level of 0.05 was used as the criterion for the statistical significance of all comparisons.

\section{Results}

Tissue $\mathrm{pO}_{2}$ alterations after burn. The changes in tissue $\mathrm{pO}_{2}$ observed at different time points post burn are shown in Fig. 1. The decrease in tissue $\mathrm{pO}_{2}$ was significant immediately $(\mathrm{P}=0.033)$ and at $6 \mathrm{~h}(\mathrm{P}=0.004)$ as compared to the control hind limbs, and returned to the initial tissue $\mathrm{pO}_{2}$ after 3 days ( $\mathrm{n}=4$ mice per group). At day 3 and thereafter, the tissue $\mathrm{pO}_{2}$ of the burned group did not differ from the controls. The tissue $\mathrm{pO}_{2}$ of the control hind limb was constant throughout the experiments.

Burn injury affects the tissue redox status as measured by $E P R$. The results obtained for redox measurements are shown in Fig. 3. The control mice had the same skeletal muscle redox status on day 0 and 3 . Also, no significant difference was found in the decay rates of the nitroxide at day 0 (immediately post burn) and day 3 between the control and burn groups. The decay rate of the nitroxide in the burn group at $6 \mathrm{~h}(0.000604 \pm$ $\left.0.00008 \mathrm{sec}^{-1}\right)$ differed significantly from the decay rate in the control group at day $0\left(0.00165 \pm 0.00024 \mathrm{sec}^{-1}, \mathrm{P}=0.027\right)$ and from the burn group at time $0\left(0.00143 \pm 0.00151 \mathrm{sec}^{-1}\right.$, $\mathrm{P}=0.018, \mathrm{t}$-test).

ROS production after burn. We measured ROS production with EPR in vivo using of the oxidation of hydroxylamine (CP-H). Before burn (baseline), a low-intensity EPR signal was detectable from the mouse hind limb. Twelve hours after burn, the oxidized $\mathrm{CP}-\mathrm{H}$ signal increased, indicating an increase in ROS generation by the burn. The oxidized CP-H signal increased between 12 and $24 \mathrm{~h}$, suggesting a further increase in ROS formation (Fig. 4). No such increase in the EPR signal of the nitroxide was observed in the control group at all time points.

Expression of antioxidant genes after burn. In our transcriptome studies, we compared the expression of all genes that 
A

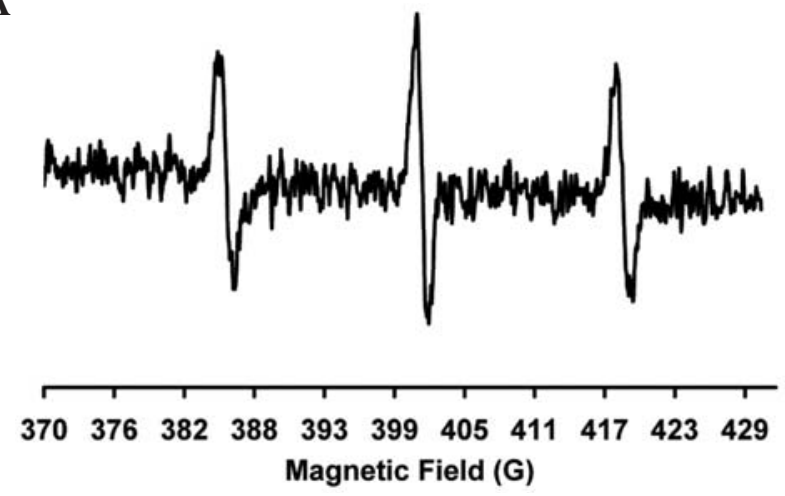

B

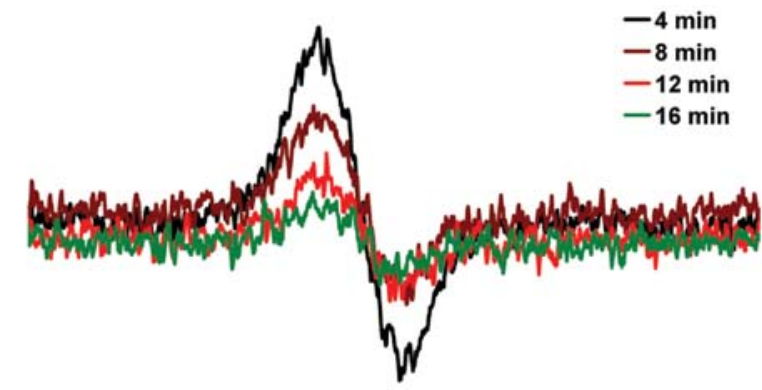

Magnetic Field (G)

Figure 2. (A) Typical in vivo EPR spectra of the CPA (3-carbamoyl-2,2,5,5-tetramethylpyrrolidin-1-yloxyl free radical, $150 \mathrm{mg} / \mathrm{kg}$ ) nitroxide injected into mice through the tail vein. The spectra were collected over the gastrocnemius muscle of the mice using a 1.2-GHz EPR spectrometer equipped with an external loop resonator. (B) The middle component of the EPR spectrum was used to follow the reduction of the nitroxide over time to determine tissue redox status.

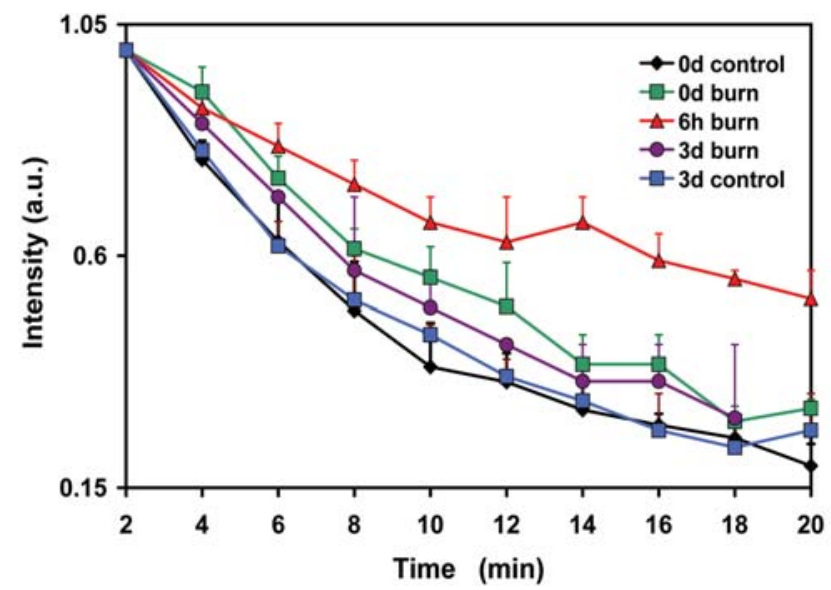

Figure 3. The decay kinetics of the nitroxide (CPA) in the gastrocnemius muscle of the mice in control and burn groups. The redox status of the control group was measured at day $0(0 \mathrm{~d})$ and day $3(3 \mathrm{~d})$. The redox status of the burn group was measured at day $0,6 \mathrm{~h}$ and day 3 . Values are means $\pm \mathrm{SE}$; $\mathrm{n}=4$.

might lead to metabolic dysfunction in skeletal muscle after burn. We found the differential expression of three metabolic genes at 3 time points following a localized, but severe, hind limb burn (Table I). Slc25a4 or Ant1 gene expression was downregulated at 1 and 3 days post burn, Sod 2 expression was downregulated at day 1 , and the expression of Gpx 1 was moderately upregulated at 3 days post burn.

\section{Discussion}

In vivo EPR was successfully used to investigate changes in tissue $\mathrm{pO}_{2}$, redox state and ROS generation after burn injury. The downregulation of the antioxidant genes Ant 1 and Sod2 in skeletal muscle, which parallels the rise of mitochondrial ROS as detected by in vivo EPR, suggests that insufficient oxidative defense following local burn trauma may lead to mitochondrial dysfunction via mtDNA oxidative damage due to ROS toxicity. Our findings suggest that tissue $\mathrm{pO}_{2}$, redox state and ROS by EPR in skeletal muscle could serve as markers of metabolic changes for following the pathophysiology of burn trauma and associated oxidative damage.

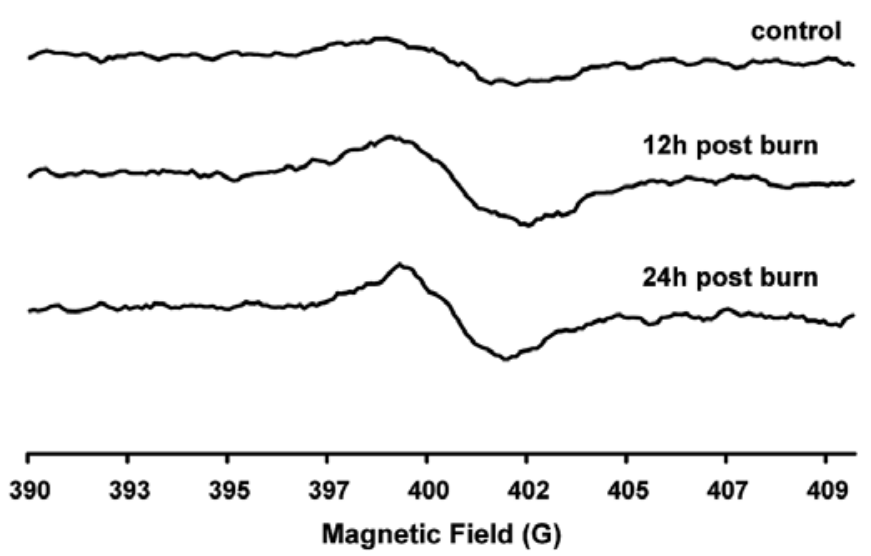

Figure 4. Typical in vivo EPR spectra of the oxidized CPH spin trap injected via the tail vein, and the spectra collected over the gastrocnemius tissue of the mice pre-, $12 \mathrm{~h}$ and $24 \mathrm{~h}$ post burn trauma. The oxidation of the hydroxylamine to the nitroxide occurs due to its oxidation by ROS in the tissue.

The principal findings of the present study are complementary to findings by NMR, suggesting that a major factor in the progression of mitochondrial skeletal muscle dysfunction in burns results from defects in oxidative phosphorylation (OXPHOS) $(22-24,33,34)$. Indeed, findings with the same mouse 'local' burn model (affecting the hind limb and representing $3-5 \%$ of total body surface area) demonstrated that burn injury causes a significant dysregulation in OXPHOS and a decrease in the ATP synthesis rate (23). Taken together, NMR and the present EPR oximetry data suggest that the decrease in ATP synthesis rate at day 3 previously observed following burn trauma (23) might be preceded by a significant reduction in tissue $\mathrm{pO}_{2}$ at $6 \mathrm{~h}$, as observed in this study. Of note, the ATP synthesis rate remains significantly low, even after tissue $\mathrm{pO}_{2}$ have normalized, which indicates that the dysregulation of OXPHOS in burn trauma is not tissue $\mathrm{O}_{2}$ dependent. On the other hand, the dysregulation of OXPHOS in burn trauma at $24 \mathrm{~h}$ and thereafter is preceded by the downregulation of a key transcription factor, the peroxisome proliferator-activated receptor $\gamma$ coactivator 1 , or PBC-1ß (23). These are hypothesized to play a central role in regulating 
Table I. Differential expression of selected antioxidant genes at $6 \mathrm{~h}$, day 1 and day 3 post burn trauma.

\begin{tabular}{|c|c|c|c|c|c|}
\hline GenBank accession no. & Product & Gene symbol & $6 \mathrm{~h}$ & Day 1 & Day 3 \\
\hline AV327862 & $\begin{array}{l}\text { Solute carrier family } 25 \\
\text { (mitochondrial carrier; adenine } \\
\text { nucleotide translocator), member } 4\end{array}$ & Slc $25 \mathrm{a} 4$ or Ant 1 & I & -1.41 & -1.61 \\
\hline AW701319 & $\begin{array}{l}\text { Superoxide dismutase } 2 \text {, } \\
\text { mitochondrial }\end{array}$ & Sod2 & I & -1.72 & l \\
\hline BI219063 & Glutathione peroxidase 1 & Gpx1 & / & / & +1.81 \\
\hline
\end{tabular}

Values represent the relative expression intensity in burn animals versus normal control animals. (/) not differentially expressed compared to controls.

energy homeostasis and metabolism (35) at $6 \mathrm{~h}$ post burn (23), which coincides with significant changes in EPR-derived parameters at the same time point as shown in this study. This downregulation of PBC-1ß by $6 \mathrm{~h}$ post burn probably causes the downregulation of the genes involved in OXPHOS (23) and in detoxification, mainly Ant1 and Sod2 (Table I), since the expression of such genes is downregulated by $24 \mathrm{~h}$ post burn. As a result of this dysregulation, the balance of detoxification and ROS generation, which has been detected as accumulating as early as $12 \mathrm{~h}$ post burn, is prevented. To this end, the present study confirms the notion that certain components of the ROS scavenging pathway are linked by the PBC-1ß to mitochondrial OXPHOS, apparently enabling or disabling cells to maintain normal redox status in response to changing oxidative capacity (36).

Another major finding of this study is the effect of the burn on skeletal muscle redox status, which coincided with decreased expression in two major antioxidant systems; that is, a decrease in Ant1 and Sod2 gene expression, and a moderate increase in Gpx1 gene expression. The mitochondria are one of the major sources of endogenous ROS in the cell, including superoxide anions $\left(\mathrm{O}_{2}{ }^{--}\right)$, hydrogen peroxide $\left(\mathrm{H}_{2} \mathrm{O}_{2}\right)$, and hydroxyl radicals $\left(\mathrm{OH}^{\bullet}\right)$ (37). Since $\mathrm{OH}^{\bullet}$ is largely limited by diffusion, it is more likely to cause damage directly to the mitochondria, especially to the inner membrane where they are generated. Additionally, ROS can initiate damage to nucleic acids, proteins and lipids $(38,39)$. Since mitochondrial DNA (mtDNA) is located closer to the site of ROS generation, lacks protective histones and has more limited base excision repair mechanisms than the nucleus, it is more vulnerable to oxidative damage than nuclear DNA (nDNA) $(40,41)$. Moreover, due to its proximity to OXPHOS in the mitochondrial inner membrane, mtDNA has been hypothesized to be a major target for ROS damage. Hence, inhibition of OXPHOS is predicted to result in the progressive destruction of mtDNA. Since OXPHOS is significantly inhibited in skeletal muscle post burn $(23,24)$, we hypothesized that the mitochondria should produce higher levels of ROS post burn. This has been confirmed in this study. The increased ROS should induce antioxidant defenses and prevent damage to mitochondrial macromolecules; in the absence of such defenses oxidative damage ensues. Based on these findings, we suggest that increased ROS results in oxidative damage of mitochondrial macromolecules, possibly including mtDNA. Indeed, this is what happens in Ant1 knockout mice, the mitochondria of which produce increased levels of ROS and show a striking increase in the accumulation of mtDNA rearrangements (42). In agreement with this, increased mitochondrial oxidative stress is reported in mice lacking the glutathione peroxidase-1 gene (43).

In aging, where we also have mitochondrial dysfunction, reports have indicated that somatic mtDNA mutations cause OXPHOS dysfunction without affecting antioxidant systems and ROS production, and that OXPHOS dysfunction is the primary inducer of premature aging in mtDNA-mutated mice (44). Others, however, have reported that electron leakage from the electron transport chain causes specific damage to their subunits and increased ROS generation as oxidative damage accumulates (45). This leads to further mitochondrial dysfunction in a cyclical process, which underlies the progressive physiological decline of the aged mouse heart (45). In another study, the DNA damage induced by acrolein in HepG2 cells was related to oxidative stress produced as a result of increased ROS and the depletion of glutathione (GSH) $(45,46)$. Studies in heart muscle suggest that antioxidant vitamin therapy following burn trauma provides at least partial cardioprotection by inhibiting translocation of the transcription factor $\mathrm{NF}-\kappa \mathrm{B}$ and by interrupting cardiac inflammatory cytokine secretion (47). Additional studies by the same research group support the hypothesis that oxidative stress critically influences the heart following burn injury, suggesting that antioxidant strategies designed to either inhibit free radical formation or to scavenge free radicals may provide organ protection in patients with burn injury (48). To date, there have been no studies that stimulated mitochondrial production of ROS or measured the resulting ROS and/or bioenergetic and oxidative damage in the skeletal muscle.

Finally, our results indicate that EPR is a promising tool for making an in vivo assessment of the consequences of burn injury in well-established burn injury models. We also propose that it is complementary to NMR, which has already shown the consequences of bioenergetic and mitochondrial dysfunction in vivo (22-25). Thus, our results lead us to conclude that bioenergetic dysfunction and insufficient oxidative defense following local burn trauma may lead to oxidative damage. In our future multidisciplinary studies, we plan to test this hypothesis, to potentially assign physiological functions to 
key regulatory genes, and to demonstrate whether these genes are involved in burn injury. These insights will lead to new therapeutic strategies for the prevention of potentially fatal complications in burn patients. We also believe that our findings may be clinically relevant in molecular medicine.

\section{Acknowledgements}

This work was supported in part by a Shriners Hospital for Children research grant no. 8893 to A. Aria Tzika, a National Institutes of Health (NIH) Center Grant P50GM021700 (Ronald G. Tompkins, PI; A. Aria Tzika, Director of NMR Core), and by a National Institute of Diabetes and Digest and Kidney (NIDDK) research grant no. DK072112 to Nadeem Khan. We thank Dr Huagang Hou, Dartmouth Medical School, for advice on the procedures for EPR measurements. We also thank Drs Katie Edmondson and Ann Power Smith of Write Science Right for editorial assistance.

\section{References}

1. Gallez B and Swartz HM: In vivo EPR: when, how and why? NMR Biomed 17: 223-225, 2004.

2. Swartz HM, Khan N, Buckey J, et al: Clinical applications of EPR: overview and perspectives. NMR Biomed 17: 335-351, 2004.

3. Helisch A, Wagner S, Khan N, et al: Impact of mouse strain differences in innate hindlimb collateral vasculature. Arterioscler Thromb Vasc Biol 26: 520-526, 2006.

4. Khan N, Williams BB, Hou H, Li H and Swartz HM: Repetitive tissue $\mathrm{pO}_{2}$ measurements by electron paramagnetic resonance oximetry: current status and future potential for experimental and clinical studies. Antioxid Redox Signal 9: 1169-1182, 2007.

5. Towner RA, Sturgeon SA, Khan N, Hou H and Swartz HM: In vivo assessment of nodularin-induced hepatotoxicity in the rat using magnetic resonance techniques (MRI, MRS and EPR oximetry). Chem Biol Interact 139: 231-250, 2002.

6. James PE, Bacic G, Grinberg OY, et al: Endotoxin-induced changes in intrarenal $\mathrm{pO}_{2}$, measured by in vivo electron paramagnetic resonance oximetry and magnetic resonance imaging. Free Radic Biol Med 21: 25-34, 1996.

7. Swartz HM, Khan N and Khramtsov VV: Use of electron paramagnetic resonance spectroscopy to evaluate the redox state in vivo. Antioxid Redox Signal 9: 1757-1771, 2007.

8. Khan N and Swartz H: Measurements in vivo of parameters pertinent to ROS/RNS using EPR spectroscopy. Mol Cell Biochem 234: 341-357, 2002.

9. Khan N, Williams B and Swartz H: Clinical applications of in vivo EPR: rationale and initial results. Appl Magn Reson 30: 185-199, 2006.

10. Szczepaniak LS, Babcock EE, Schick F, et al: Measurement of intracellular triglyceride stores by ${ }^{1} \mathrm{H}$ spectroscopy: validation in vivo. Am J Physiol 276: E977-E989, 1999.

11. Kuhlmann J, Neumann-Haefelin C, Belz U, Kramer W, Juretschke HP and Herling AW: Correlation between insulin resistance and intramyocellular lipid levels in rats. Magn Reson Med 53: 1275-1282, 2005.

12. Boesch C, Slotboom J, Hoppeler H and Kreis R: In vivo determination of intra-myocellular lipids in human muscle by means of localized 1H-MR-spectroscopy. Magn Reson Med 37: 484-493, 1997.

13. Schick F, Eismann B, Jung WI, Bongers H, Bunse M and Lutz O: Comparison of localized proton NMR signals of skeletal muscle and fat tissue in vivo: two lipid compartments in muscle tissue. Magn Reson Med 29: 158-167, 1993.

14. Neumann-Haefelin C, Kuhlmann J, Belz U, et al: Determinants of intramyocellular lipid concentrations in rat hindleg muscle. Magn Reson Med 50: 242-248, 2003.

15. Hwang JH, Pan JW, Heydari S, Hetherington HP and Stein DT: Regional differences in intramyocellular lipids in humans observed by in vivo ${ }^{1} \mathrm{H}-\mathrm{MR}$ spectroscopic imaging. J Appl Physiol 90: 1267-1274, 2001.

16. Krssak M, Petersen KF, Dresner A, et al: Intramyocellular lipid concentrations are correlated with insulin sensitivity in humans: a 1H NMR spectroscopy study. Diabetologia 42: 113-116, 1999.
17. Krssak M, Petersen KF, Bergeron R, et al: Intramuscular glycogen and intramyocellular lipid utilization during prolonged exercise and recovery in man: a $13 \mathrm{C}$ and $1 \mathrm{H}$ nuclear magnetic resonance spectroscopy study. J Clin Endocrinol Metab 85: 748-754, 2000.

18. Jacob S, Machann J, Rett K, et al: Association of increased intramyocellular lipid content with insulin resistance in lean nondiabetic offspring of type 2 diabetic subjects. Diabetes 48 : 1113-1119, 1999.

19. Goodpaster BH, He J, Watkins S and Kelley DE: Skeletal muscle lipid content and insulin resistance: evidence for a paradox in endurance-trained athletes. J Clin Endocrinol Metab 86: 5755-5761, 2001.

20. Anderwald C, Bernroider E, Krssak M, et al: Effects of insulin treatment in type 2 diabetic patients on intracellular lipid content in liver and skeletal muscle. Diabetes 51: 3025-3032, 2002.

21. Sinha R, Dufour S, Petersen KF, et al: Assessment of skeletal muscle triglyceride content by (1) H nuclear magnetic resonance spectroscopy in lean and obese adolescents: relationships to insulin sensitivity, total body fat, and central adiposity. Diabetes 51: 1022-1027, 2002.

22. Astrakas LG, Goljer I, Yasuhara S, et al: Proton NMR spectroscopy shows lipids accumulate in skeletal muscle in response to burn trauma-induced apoptosis. FASEB J 19: 1431-1440, 2005.

23. Padfield KE, Astrakas LG, Zhang Q, et al: Burn injury causes mitochondrial dysfunction in skeletal muscle. Proc Natl Acad Sci USA 102: 5368-5373, 2005.

24. Tzika AA, Mintzopoulos D, Padfield K, et al: Reduced rate of adenosine triphosphate synthesis by in vivo ${ }^{31} \mathrm{P}$ nuclear magnetic resonance spectroscopy and downregulation of PGC-1ß in distal skeletal muscle following burn. Int J Mol Med 21: 201-208, 2008.

25. Tzika AA, Astrakas LG, Cao H, et al: Murine intramyocellular lipids quantified by NMR act as metabolic biomarkers in burn trauma. Int J Mol M ed 21: 825-832, 2008.

26. Laird NM and Ware JH: Random-effects models for longitudinal data. Biometrics 38: 963-974, 1982.

27. Tomera JF and Martyn J: Systemic effects of single hindlimb burn injury on skeletal muscle function and cyclic nucleotide levels in the murine model. Burns Incl Therm Inj 14: 210-219, 1988.

28. Dunn J and Swartz H: In vivo electron paramagnetic resonance oximetry with particulate materials. Methods 30: 159-166, 2003.

29. He G, Kutala VK, Kuppusamy P and Zweier JL: In vivo measurement and mapping of skin redox stress induced by ultraviolet light exposure. Free Radic Biol Med 36: 665-672, 2004.

30. Ilangovan G, Li H, Zweier JL and Kuppusamy P: In vivo measurement of tumor redox environment using EPR spectroscopy. Mol Cell Biochem 234: 393-398, 2002.

31. Liu S, Timmins GS, Shi H, Gasparovic CM and Liu KJ: Application of in vivo EPR in brain research: monitoring tissue oxygenation, blood flow, and oxidative stress. NMR Biomed 17: 327-334, 2004.

32. Vittinghoff E, Glidden D, Shiboski S and McCulloch C: Regression Methods in Biostatistics. Linear, Logistic, Survival, and Repeated Measures Models. Springer, New York, 2005.

33. Padfield KE, Zhang Q, Gopalan S, et al: Local and distant burn injury alter immunoinflammatory gene expression in skeletal muscle. J Trauma 61: 280-292, 2006.

34. Zhang Q, Cao H, Astrakas LG, et al: Uncoupling protein 3 expression and intramyocellular lipid accumulation by NMR following local burn trauma. Int J Mol Med 18: 1223-1229, 2006.

35. Handschin C and Spiegelman BM: Peroxisome proliferatoractivated receptor gamma coactivator 1 coactivators, energy homeostasis, and metabolism. Endocr Rev 27: 728-735, 2006.

36. Lin J, Handschin C and Spiegelman BM: Metabolic control through the PGC-1 family of transcription coactivators. Cell Metab 1: 361-370, 2005.

37. Wallace DC and Melov S: Radicals r'aging. Nat Genet 19: 105-106, 1998.

38. Cadenas E and Davies KJ: Mitochondrial free radical generation, oxidative stress, and aging. Free Radic Biol Med 29: 222-230, 2000 .

39. Szeto HH: Mitochondria-targeted peptide antioxidants: novel neuroprotective agents. AAAPS J 8: E521-E531, 2006.

40. Bandy B and Davison AJ: Mitochondrial mutations may increase oxidative stress: implications for carcinogenesis and aging? Free Radic Biol Med 8: 523-539, 1990. 
41. Imam SZ, Karahalil B, Hogue BA, Souza-Pinto NC and Bohr VA: Mitochondrial and nuclear DNA-repair capacity of various brain regions in mouse is altered in an age-dependent manner. Neurobiol Aging 27: 1129-1136, 2006.

42. Esposito LA, Melov S, Panov A, Cottrell BA and Wallace DC: Mitochondrial disease in mouse results in increased oxidative stress. Proc Natl Acad Sci USA 96: 4820-4825, 1999.

43. Esposito LA, Kokoszka JE, Waymire KG, Cottrell B, MacGregor GR and Wallace DC: Mitochondrial oxidative stress in mice lacking the glutathione peroxidase-1 gene. Free Radic Biol Med 28: 754-766, 2000.

44. Trifunovic A, Hansson A, Wredenberg A, et al: Somatic mtDNA mutations cause aging phenotypes without affecting reactive oxygen species production. Proc Natl Acad Sci USA 102: 17993-17998, 2005.
45. Choksi KB and Papaconstantinou J: Age-related alterations in oxidatively damaged proteins of mouse heart mitochondrial electron transport chain complexes. Free Radic Biol Med 44: 1795-1805, 2008.

46. Li L, Jiang L, Geng C, Cao J and Zhong L: The role of oxidative stress in acrolein-induced DNA damage in HepG2 cells. Free Radic Res 42: 354-361, 2008.

47. Horton JW, White DJ, Maass DL, Hybki DP, Haudek S and Giroir B: Antioxidant vitamin therapy alters burn trauma-mediated cardiac NF-kappaB activation and cardiomyocyte cytokine secretion. J Trauma 50: 397-407, 2001.

48. Horton JW: Free radicals and lipid peroxidation mediated injury in burn trauma: the role of antioxidant therapy. Toxicology 189: 75-88, 2003. 